\title{
The Mechanism of the Earthquakes in Terms of the Dislocation Theory
}

\author{
S. Droste-R. Teisseyre
}

During the last years there originated quite a number of model theories of seismic foci. In such models the dynamic proces in the focus is substituted by the action of a classic system of equivalent mass forces. Among works on this subject the publications of the school of W. I. Keylis-Borok, as well as of $\mathrm{H}$. Honda should be mentioned in the first order.

Let us consider the simplest model of a focus, that is the case of a simple point force. The expression for displacement $\bar{u}$ in such a case is given by the classic Love's formulas. The dependence of the force from time is here expressed by an arbitrary function $\varkappa(t)$. If this function will be discontinuous, as for example in case of a step function $(\varkappa=0$ for $t<0, \varkappa=1$ for $t>0$ ), than Love's formula for $u$ determines the creation of a infinitesimal dislocation element at time $t=0$. This problem is discussed in detail by Nabarro $\left({ }^{10}\right)$.

In this manner model theories represent the creation of dislocation elements for such cases in which the time dependence of active forces is discontinuous.

To this category there belong also the publication of A. Wwiedenskaja $\left.{ }^{12}\right)$ on the mechanism of earthquakes, represented as a creation of a finite dislocation of the disc type. Formally her work differs from the preceding group, because of direct use of the theory of physical dislocation.

The localisation of the dislocation created - should be determined by the stresses occurring in the medium however no such relation is clearly given by the theories discussed above.

We should like to present a new theory of the mechanism of earthquakes. The starting point of this theory is the assumption, that there exists a nonhydrostatic part of stress in the medium, as well as a field of inhomogenities in the largest sense. Such inhomogenities, even very 
small in size, may serve as points of attachment. They institute the nucleus of dischargement of the strain energy occurring in the medium and together with stresses they determine the earthquake's area.

These obvious assumptions may be considered further in the light of the theory of physical dislocations, which may be interpreted as the theory of inhomogenities in a field of forces. The theory of physical dislocation was developped in the solid state physics.

Before going further on we will stop for a short while on basic definitions and consequences of the physical dislocation theory. The simplest example is represented by linear dislocation. Classically is will be defined, as foliows: a cut is executed along a chosen halfplane and afterwards the material on both sides of the cut is displaced relatively by a constant wector $\bar{b}$ (what is called Burgers' vector). The edge of the dislocated halfplane is called dislocation line.

The main part of the selfenergy of dislocation, determined as the energy of the deformation created, is acumulated in the neighbourhood of the dislocation line. The displacement of the dislocation line gives an increase in the dislocation area.

It may be said that the potential energy of physical dislocation expresses the tendency of the whole medium's deformation. This potential energy is liberated by the movement in this moment when the dislocation reaches the medium's boundary.

In order to define fully dislocation Burgers' sing convention which serves to determine the relation between the circulation along the dislocation line and the direction of relative displacement of the medium on both sides of dislocation should be used.

The dislocation vector $\bar{b}$ which is situated in the cut plane (which is called also the slip plane) may be parallel to the dislocation line in this case the dislocation is of the screw type, or it may be vertical to the dislocation line in which case we call the dislocation edge dislocation.

The external stress field exerts it's influence on the dislocation through interaction with the field of dislocation. This influence may be expressed as the action of force on an element of the dislocation line $\overline{d \sigma}$

$$
\bar{F}=(P \cdot \bar{b}) \times d \bar{\sigma}
$$

where $P$ being the stress tensor.

Similary there exist an interaction between dislocation. Dislocations of opposite signs are attracted to each other, of the same signs are being repulsed. These forces are inversely proportional to distance between them. 
Dislocation which have curved dislocation lines, especially, closed ones, will serve as a generalisation of dislocations discussed above. Dislocations having curved closed dislocation lines are called loop dislocations. We will consider now very small loop dislocations. These have interesting properties, which deserve more detailed study.

We are considering now dislocations situated in a common slip plane. Loop dislocations of the same signs are now attracted to each other. This is caused by the fact that the elements of dislocations situated nearest one to each other have opposite signs. As the result of such atraction two loop dislocations may approach each other and afterwards join into one. As a consequence of such a synthesis a dislocation comprising a greater area is created. Different kinds of dislocations may arise by means of a chain of such syntheses.

Now to the basic assumptions of the present work we will add that the value of the nonhydrostatic part of the stress field and density of inhomogenities of the medium are extrenally great in the neighbourhood of a suppossed plane $(y=0)$. As we will see this plane and it's neighbourhood constitute the stage upon which dislocative processes and accompaning earthquakes take place. This plane, together with it's neighbourhood institute the area of seismic activity. It may be called also the hypocentric plane.

Let us assume that the nonhydrostatic stresses are represented by the shear stress $p_{y z}$. Now we can proove that the constant field $p_{y z}$ on the plane may be represented by an equal distribution of loop microdislocations characterized by Burgers vector $b$, and by normal vector of the direction - y. If $n$ will represent the number of microdislocations for an unit of surface and $o_{0}$ will represent their radius, than we will obtain:

$$
p_{y z}=\frac{n b}{\varrho_{0}} \cdot \frac{\mu c^{2}}{4}\left(\frac{3}{c^{2}}-\frac{2}{a^{2}}\right) .
$$

Here we can go to the limit $n \rightarrow=\infty, b_{z} \rightarrow 0, \varrho_{0} \rightarrow 0$, but under the condition $\lim \frac{n b_{-}}{\varrho_{0}}=$ const.

Assuming the existence of inhomogenities on the considered plane, bigger loop dislocations will arise around them under the influence of the field $p_{y z}$. As an example let us consider the extreme case of inhomogenities in the forn of small incompressible intrusions. Inside the area occupied by such an intrusion no microdislocation will appear because of the incompressibility of the medium constituting this intru- 
sion. Instead there appears a bigger loop dislocation around the intrusion. In this manner inhomogenities quantize, as it were, the field $p_{y z}$. The loop dislocations created around inhomogenities we will consider as elementar loop dislocations. It may be said that elementar loop dislocations represent inhomogenities in the field $p_{y z}$. Later the action of a sufficiently great field $p_{y z}$ and the interaction between dislocations causes their deformation by an increase in surface which dislocation comprises.

Now we must assume that a fluctuation in density of inhomogenities exist on the considered plane. It could be now said also in other words that there exist a fluctuation in the density of loop dislocations. In the region of the maximal concentration of inhomogenities, there arise a greater dislocation by it's own growth and by syntheses with neigh-

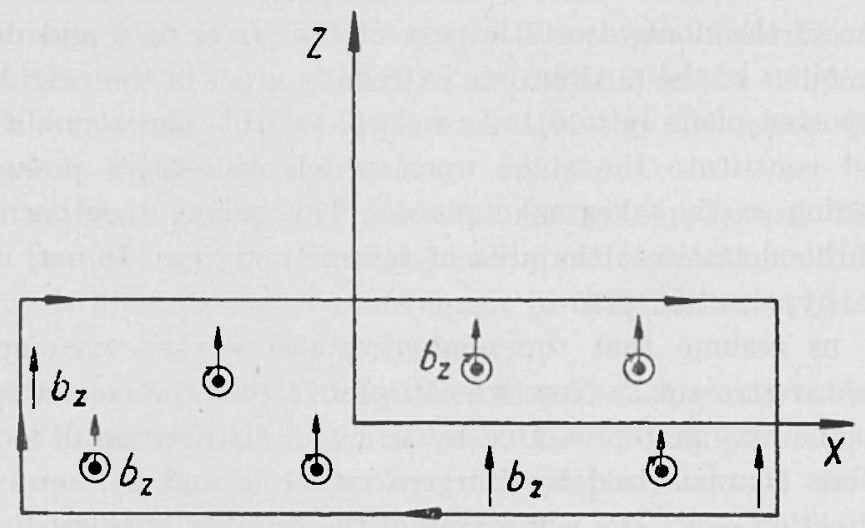

Fig. 1.

bouring dislocation. The creation of greater dislocation elements may be of violent nature giving source to seismic shocks. It should be mentioned, however, that the greater part of the work of the field $p_{y z}$ is consumed by an incorease in internal energy of the dislocation. Therefore it is to note, the earthquakes caused by violent creation of dislocations or of their movements will belong to the weaker category. The dislocation created in the manner described above may be represented by a pair of edge dislocations limited by sides of screw dislocations (fig. 1). In an extreme case we obtain a pure pair of edge dislocations infinitely long, composed with two dislocation of opposite signs.

Further under the influence of the field $p_{y z}$ the dislocation $\mathrm{1}^{+}$and $\mathrm{1}^{-}$ go asunder. This may occur under the condition that, the forces of the 
field $p_{y z}$ must be greater, than the force of attraction, between the pair of dislocations (forces are usually expresses for an unit of length).

We will now pay attention to particular cases of dislocative processes on the discussed plane. Firstly we will examine the case when there exists one center of generation of dislocations. Let us assume that it is concentrated along the line " 0 " (fig. 2).

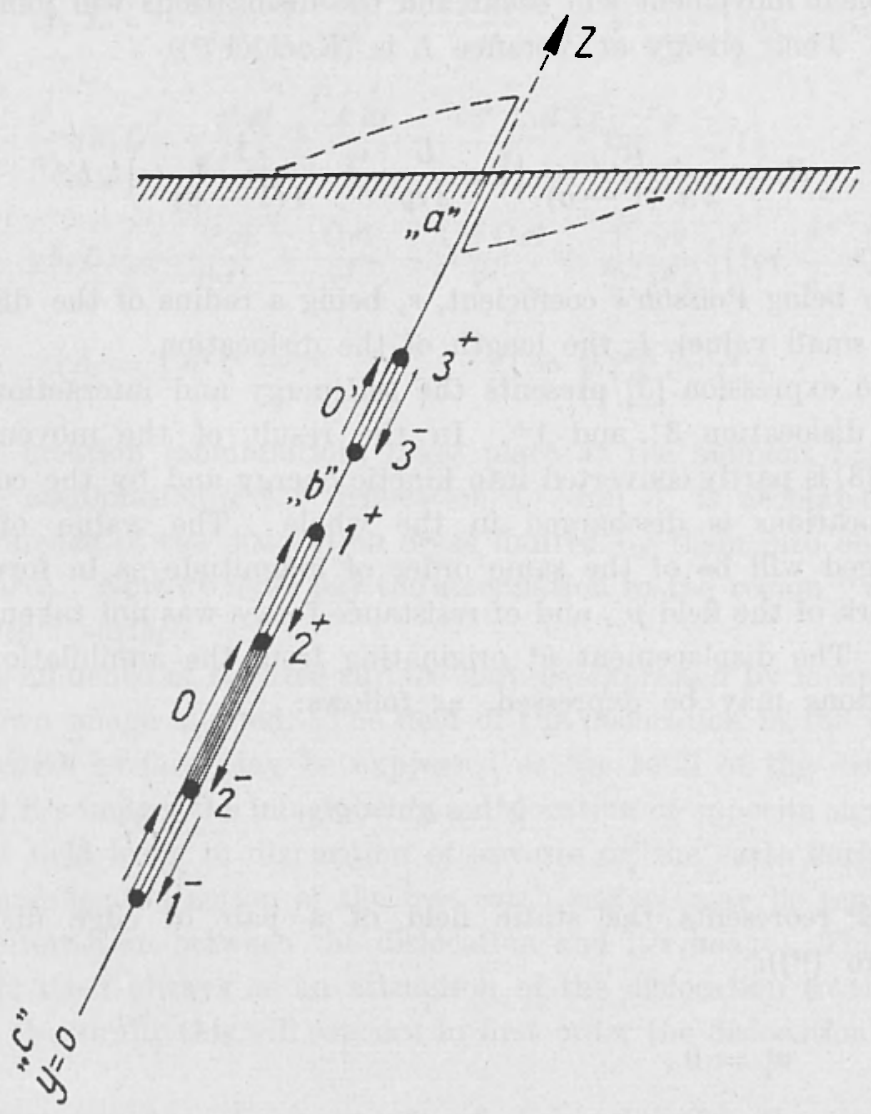

Fig. 2.

Many pairs of dislocations are created around the line " $0 "$ as the result of constant action of the field $p_{y z}$. Each of these pairs go asunder, as it was described above. In consequence a situation as on drawing is created. In such case the neighbouring dislocation $1^{+}$and $2^{+}$are of the same sign and are repulsing each other. This leads, as it were, to " jostling" of the extremely situated dislocation by dislocations situated near centre " 0 " towards external directions. 
When there exists also the second center " 0 " " of generation of the dislocation $\left(3^{+}, 3^{-}\right)$, then the dislocation $1^{+}, 3^{-}$will attract and approach to each other.

In the area (" $b$ ") the dislocations $3^{-}$and $1^{+}$will join and annihilate themselves, with accompanying dischargement of their energy. We assume that initially the approach is slow, when they reach the distance $L$ a violent movement will occur and the dislocations will join and disapear. Their energy at distance $L$ is (Koehler $\left({ }^{3}\right)$ )

$$
E=\frac{\mu}{2 \pi(1-\sigma)}\left(\log \frac{L}{2 r_{0}}-\frac{1}{4(1-\sigma)}\right) l_{0} b_{z}^{2}
$$

where $\sigma$ being Poisson's coefficient, $r_{o}$ being a radius of the dislocation line (a small value), $l_{o}$ the length of the dislocation.

The expression [3] presents the selfenergy and interaction energy of the dislocation $3^{-}$and $1^{+}$. In the result of the movement the energy [3] is partly converted into kinetic energy and by the confluance of dislocations is discharged in the whole. The value of energy discharged will be of the same order of magnitude as in formule [3]. The work of the field $p_{y z}$ and of resistance forces was not taken into account. The displacement $\bar{u}^{a}$ originating from the annihilation of the dislocations may be expressed, as follows:

$$
\bar{u}^{a}=\bar{u}^{s}-\bar{u}^{c}
$$

where $u^{\text {s }}$ reoresents the static field of a pair of edge dislocations (Nabarro $\left.{ }^{(10}\right)$ ):

$$
\begin{aligned}
u_{x}^{s}= & 0 \\
u_{y}^{s}= & \frac{1}{\pi} K \frac{y^{2} z}{r^{4}} b_{z} L+\frac{1}{2 \pi}(1-K) \frac{z}{r^{2}} b_{z} L, \\
u_{s}= & \frac{1}{\pi} K \frac{y z^{2}}{r^{4}} b_{z} L+\frac{1}{2 \pi}(1-K) \frac{z}{r^{2}} b_{z} L, \\
& \left(K=1-\frac{c^{2}}{a^{2}}, r^{2}=y^{2}+z^{2}\right)
\end{aligned}
$$


and $u^{c}$ represents the field of creation of a pair of edge dislocations $\left({ }^{13}\right)$ :

$$
\begin{aligned}
& u_{x}^{c}-0 \text {, } \\
& u_{y}^{c}=\frac{1}{\pi} \frac{c^{2}}{a^{2}}, n_{-} \tau_{1}\left(-\frac{y^{2} a t}{r^{4} A}+\frac{A a t}{r^{4}}-\frac{4 y^{2} A a t}{r^{6}}\right)\left(\text { for } \frac{r}{a} \leqslant t\right)- \\
& -\frac{1}{\pi} z \dot{v}_{z} L\left(-\frac{y^{2} c t}{r^{4} C}+\frac{C c t}{r^{4}}-\frac{4 y^{2} C c t}{r^{6}}+\frac{1}{2} \frac{c t}{r^{2} C}\right)\left(\text { for } \frac{r}{c} \leqslant t\right) \\
& u_{x}^{c}=\frac{1}{\pi} \frac{c^{2}}{a^{2}} y \dot{u}_{z} L\left(-\frac{z^{2} a t}{r^{4} A}+\frac{A a t}{r^{4}}-\frac{4 z^{2} A a t}{r^{2}}\right)\left(\text { for } \frac{r}{a} \leqslant t\right)- \\
& -\frac{1}{\pi} z_{3}^{2}=\left(-\frac{z^{2} c t}{r^{4} C}+\frac{C c t}{r^{2}}-\frac{4 z^{2} C c t}{r^{8}}+\frac{1}{2} \frac{c t}{r^{2} C}\right)\left(\text { for } \frac{r}{c} \leqslant v\right) \\
& \left(A=\sqrt{a^{2} t^{2}-r^{2}} \quad, \quad O=\sqrt{c^{2} t^{2}-r^{2}}\right) .
\end{aligned}
$$

The creation (annihilation) takes place at the moment $t=0$.

The annihilation of the dislocation $1^{+}$and $3^{-}$is accompanied by the confluence of the dislocation areas limited by them into one deformation area. Now we must pay the attenuation to the region " $a$ " near the Earth's surface.

The influence of the free surface may be expressed by means of the well known image method. The field of the dislocation in the presence of free earth surface may be expressed as the total of the dislocation field and it's image, the image being a dislocation of opposite sign. Such resultant field leads to disparition of stresses on the earth surface. In such a manner the action of the free earth surface may be represented as the interaction between the dislocation and it's image. This action manifests itself always as an attraction of the dislocation to the earth surface. Naturally this will concern in first order the dislocation $3^{+}$and after $2^{+}$.

In consequence of movements of dislocation $3^{+}$the dislocation area approach to the Earth's surface. When the dislocation reaches it, then it's stresses will be released. As in this case the field of stresses equals the total of the dislocation and it's image, the energy of dischargement will be expressed as in the former case but with a coefficient equals to $1 / 2$

$$
E \doteq \frac{1}{2} \frac{\mu}{2 \pi(1-\sigma)}\left(\log \frac{L}{r_{0}}-\frac{1}{4(1-\sigma)}\right) l_{0} b_{z}^{2}
$$


where $L$ represents now the distance between the Earth's surface and the dislocation at this moment, when the rapid movement of the dislocation in the direction of the surface begins. One would say, at this moment when the dislocation is about to leap to the earth surface.

In the area " $c$ " the dislocation $2^{-}$moves deep into the Earth accompanied by the deformation of the medium. In such a manner the slip increases also in this direction. It may be postulated that in deep strata of the Earth there will also occur a dischargement of dislocation stresses. The character of this dischargement, however, may be more gradual and it is difficult to say something precise about it at this moment.

I will amplify the above, presenting a few data concerning the earthquakes of type " $a$ " that is to say of this type in which the dischargement of energy take place on the Earth's surface and the deformations represented by dislocations reach this surface.

For example let us consider several great earthquakes for which the length and magnitude of slip of dislocations on the earth surface are known (Table 1).

The mechanism of earthquakes presented above constitutes at the same time a theory of origin of dislocations. From the above considerations follows that they originate as the result of displacement of physical dislocations along a given plane. The physical dislocations are connected closely with the existance of stresses and inhomogenities of the media.

Table 1

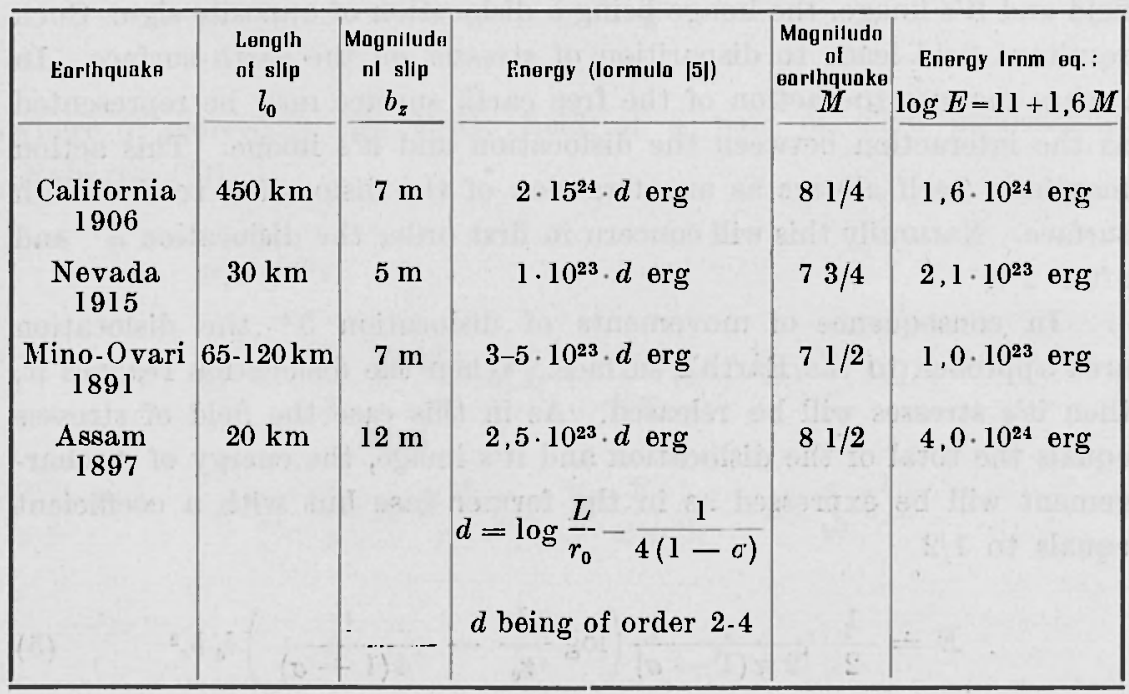


To the end of this communication we shoud like to add that the field $p_{y z}$ acting in the neighbourhood of the plane $y=0$ characterized by great inhomogenities, acts also on neighbouring elements of the surfaces $z=$ const. The processes occurring in these planes will be of course less important and may be considered as only accompaning the basic phenomena along the plane $y=0$.

\section{RIASSUNTO}

Viene esposto un nuovo meccanismo per terremoti, alla luce della. teoria delle dislocazioni. I presupposti base sono l'esistenza di una parte non idrostatica delle tensioni nel mezzo $\left(p_{y^{*}}\right)$ e una certa distribuzione delle inomogeneità. Questa distribuzione determina un piano e il suo intorno, come il piano del processo di dislocazione attiva (piano di slittamento, $y=0)$.

Si dimostra che le inomogeneita possono essere rappresentate da dislocazioni a cappio, in presenza del campo $p_{y z}$. Le dislocazioni a cappio possono aumentare sotto l'influsso del campo $p_{y z}$ e sotto l'interazione fra le medesime (sintesi dei cappi). Come risultato, conseguono coppie di dislocazioni torcenti e di confine. Allora le dislocazioni della stessa coppia sono costrette, dall'intenso campo $p_{y z}$, a procedere separatamente. L'area di scorrimento, in tal modo, aumenta. La fusione delle due dislocazioni associate alle coppie vicine, deternami il loro annientamento e la liberazione della loro energia, il che provoca un ulteriore aumento dell'area di scorrimento omogeneo. Similmente, quando la dislocazione raggiunge la superficie terrestre, la sua energia verrà liberata (nel qual caso, va preso in considerazione il fattore 1/2 nei confronti con il caso precedente).

Il confronto dell'energia liberata, così determinata, è in buon accordo con $i$ dati di osservazione.

\section{$A B S T R A C T$}

A new mechanism of earthquakes is evolved in the light of the physical dislocation theory. The basic assumptions are the existence of a nonhydrostatic part of stresses in the medium $\left(p_{y z}\right)$ and a supposed distribution of inhomogenities. This distribution determines a given plane and it's neighbourhood, as the plane of active dislocation processes (slip plane, $y=0$ ). 
It is shown that the inhomogenities could be represented by the loop dislocations in the presence of the field $p_{y z}$. The loop dislocations may increase under the influence of the field $p_{y_{z}}$ and under the interaction between themselves (synthesis of loops). As the result, there arise pairs of the screw as well as edge dislocations. Then the dislocations from the same pair are constrained by the strong field $p_{y z}$ to go asunder. In such manner the slip area increases. The blending of the two dislocations from the neighbouring pairs give their annihilation and dischargement of their energy, this is accompanied by further increase in the homogeneus slip area. Similarly when the dislocation reaches the Earth's surface it's energy will be discharged (now the factor 1/2 in the comparison with the preceding case must be taken into account).

The comparison of the dischargement's energy is in good agreement with the observation data.

\section{REFERENCES}

(1) Bilby, B. A., Proc. Phys. Soc. A., 63, 191 (1950).

(2) Burgers, I. M., Proc. Acad. Sci. Amst., 42, 293 (1939).

(3) Eshelby, I. D., Proc. Roy. Soc. A., 197, 396 (1949).

(4) - Phil. Trans. Roy. Soc. Lond. A., 244, 877, 87 (1951).

(5) - Frank, F. C., Nabarro, F. R. N., Phil. Mag., 41, 351 (1951).

( $\left.{ }^{8}\right)$ Frank, F. C., Proc. Phys. Soc. A., 62, 131 (1949).

(7) Honda, H., Sci. Rep. Tohoku Univ., Ser. 5, Geophys. 9, Supp. (1957).

(8) KEYLIS-BoRoK, W. I., D. A. N. USSR, 70, 6 (1950).

(甲) Koeirler, I. C., Plys. Rev., 60, 397 (194).

(10) Nabarro, F. R. N., Phil. Mag., 122, 1224 (1951).

(i1) Peach, M., Koehler, I. C., Phys. Rev., 80, 436 (1950).

(12) Wwiedenskaja, A. W., Izw. A. N. USSR, 3, 277 (1956).

(13) Teisseyre, R., Acta Geophysica Polonica (in press). 